\title{
The Kinetics and Feedback Inhibition of Cytidine 5'-Triphosphate Synthetase in Wild-Type and Mutant Chinese Hamster Cells
}

\author{
John D. McLaren ${ }^{1}$ and Ernest H. Y. Chu ${ }^{1,2}$ \\ Received 26 Oct. 1983-Final 20 Apr. 1984
}

The kinetics and cytidine 5'-triphosphate (CTP) feedback inhibition of CTP synthetase in wild-type and four mutants of Chinese hamster V79 cells have been studied. The enzymes of the wild type and three of the four mutants exhibited positive cooperativity with the substrate uridine 5'-triphosphate $(U T P)$. Three of the mutants had $\mathrm{K}_{\mathrm{m} \text { app }}$ and $\mathrm{S}_{50}$ values distinctly greater than those of the wild type, while the fourth mutant had values similar to those of the wild type. All four mutants exhibited resistance to CTP feedback inhibition, while the wild type was sensitive to such inhibition. It is postulated that a single mutational event in each mutant had caused a concomitant change of the enzyme in its binding both to the substrate UTP and to the end-product CTP.

KEY WORDS: cytidine $5^{\prime}$-triphosphate (CTP); uridine 5 '-triphosphate; CTP synthetase; Chinese hamster fibroblasts; enzyme kinetics; feedback inhibition.

\section{INTRODUCTION}

In the preceding article (Chu et al., 1984) we described the isolation and initial characterization of four stable pleiotropic variants of Chinese hamster cells. All variants exhibited auxotrophy for thymidine, deoxycytidine, or deoxyuridine but not cytidine or uridine. All were resistant to uridine, 3-deazauridine, 5-fluorouridine, thymidine, and cytosine arabinoside at con-

This work was supported by Grant GM 20608 from the U.S. Public Health Service.

${ }^{1}$ Lawrence D. Buhl Center for Human Genetics, Department of Human Genetics, University of Michigan Medical School, Ann Arbor, Michigan 48109.

${ }^{2}$ To whom correspondence should be addressed. 
centrations that were toxic to the parental cells. In addition, in the variants there was a permanently enlarged pool of cytidine 5 '-triphosphate (CTP) and deoxycytidine $5^{\prime}$-triphosphate (dCTP), while the uridine $5^{\prime}$-triphosphate (UTP) pool was either reduced or remained unchanged relative to the wild-type level. This overproduction of cytidine nucleotides, together with cellular resistance to 3-deazauridine, suggests that a genetic or epigenetic change might have occurred in the variants, affecting the enzymatic conversion of UTP to CTP. It has been shown that 3-deazaUTP, a metabolite of 3-deazauridine, specifically inhibits mammalian CTP synthetase (McPartland et al., 1974; Brockman et al., 1975; McPartland and Weinfeld, 1976).

In this paper, the results of kinetic studies of CTP synthetase in the crude extracts of V79 cells and the four pleiotropic variants are presented. We demonstrate striking differences in the kinetic properties of the enzymes between the parental cells and the variants, as well as among the variants themselves. A complete disappearance of CTP feedback inhibition of the enzyme occurred in all variants.

\section{MATERIALS AND METHODS}

Materials. $\left[5,6-{ }^{3} \mathrm{H}\right]$ Uridine $5^{\prime}$-triphosphate $(35 \mathrm{Ci} / \mathrm{mmol})$ was purchased from ICN Chemical and Radioisotope Division, Irvine, Calif. The cell materials, chemicals, and methods used were the same as described in the preceding paper (Chu et al., 1984).

CTP Synthetase Assays. The assay of CTP synthetase is based on the conversion of radioactively labeled UTP to CTP, followed by the separation of substrate and product(s) by polyethyleneimine (PEI) cellulose thin-layer chromatography, as described previously (McLaren and Chu, 1983). The potential amino donors L-glutamine and $\mathrm{NH}_{4} \mathrm{HCO}_{3}$, at final concentrations of 20 and $40 \mathrm{~mm}$, respectively, were used together in each assay tube. [5,6$\left.{ }^{3} \mathrm{H}\right] \mathrm{UTP}, 2.86 \times 10^{-7} \mathrm{M}, 0.25 \mu \mathrm{Ci} / 25 \mu \mathrm{l}$ final assay volume, was the radioactive substrate. Some of the experiments used $\left[2-{ }^{14} \mathrm{C}\right]$ UTP. Nonradioactive UTP was added to each assay tube to provide the desired final UTP concentrations. Nonradioactive CTP was added to those tubes used in the determination of possible CTP inhibition of CTP synthetase. All assay tubes were incubated at $37^{\circ} \mathrm{C}$ using a temperature block. The assays were initiated by the addition of $10 \mu \mathrm{l}$ cell extract to give a final volume of $25 \mu \mathrm{l}$ in each tube.

\section{RESULTS}

Preliminary experiments with V79 cell extracts suggested that the hamster CTP synthetase did not follow simple Michaelis-Menten kinetics. A Line- 
weaver-Burk plot $(1 / v$ vs $1 / s)$ was not linear but showed a concave curve, indicating positive allosteric cooperativity. This concave curve was observed only when the UTP concentration was varied over a wide range, i.e., from approximately 0.05 to $4 \mathrm{mM}$. If the UTP concentrations were limited to approximately $0.25 \mathrm{mM}$ or greater, then the Lineweaver-Burk plot appeared to be linear (Fig. 1A). The $V_{\max }$ value is $0.67 \times 10^{-6} \mathrm{~mol} / \mathrm{liter} / \mathrm{min}$ and the $K_{m \text { app }}$ from the Lineweaver-Burk plot is $0.39 \mathrm{~mm}$ (Table I). The HanesWoolf plot ( $s / v$ vs $s$ ) of the same data (Fig. 1B) is also linear, yielding a $V_{\max }$ value of $0.65 \times 10^{-6} \mathrm{~mol} / \mathrm{liter} / \mathrm{min}$ and a $K_{m \text { app }}$ of $0.37 \mathrm{~mm}$ (Table I). In a separate experiment (data not shown) where lower UTP concentrations were used, the Hanes-Woolf plot reached a minimum ordinate value and then increased with decreasing UTP concentrations. Figure $1 \mathrm{C}$ is a Hill plot, using the $V_{\max }$ value from the Lineweaver-Burk plot (Fig. 1A). The Hill plot yields an $S_{50}$ value of $0.37 \mathrm{~mm}$ and an $n_{\text {app }}$ value of 0.92 . The $S_{50}$ value is that substrate concentration giving a velocity that is one-half the $V_{\max }$; it is not equal to the Michaelis constant, $K_{m}$, unless the $n$ value is 1.0 . The equation used is $K^{\prime}=\left[S_{50}\right]^{n}$, and for Fig. 1C the $K^{\prime}$ value is $0.70 \mathrm{mM}$.

The kinetic properties of CTP synthetase in extracts of four variant cell lines, CTPS 42, 43, 44, and 46, are graphically illustrated in Figs. 2-5 and summarized also in Table I. For all four variants we have extended the curves of the Lineweaver-Burk and Hanes-Woolf plots to intersect both the $X$ and the $Y$ axis. Since the plots for all four variants are not linear over the range of substrate concentrations used, we tried to linearize only that segment of each plot corresponding to the higher substrate concentrations. In all cases the $V_{\max }$ values obtained from the Lineweaver-Burk plots were used to calculate the values for the Hill plots. The Lineweaver-Burk plots for three of the four variants (Figs. 2A, 4A, and 5A) are concave, indicating positive allosteric cooperativity. In contrast, the Lineweaver-Burk plot for V79 cell extracts (Fig. 1A) is linear for the same UTP substrate concentrations assayed. Only at even lower UTP concentrations did the V79 enzyme show a concave curve (data not shown). In addition, the $K_{m \text { app }}$ values for the same three variants are markedly greater than that for the wild-type V79 cell extract (Table I). Finally, the Lineweaver-Burk plot for CTPS 43 (Fig. 3A) has an unconventional shape that exhibits a velocity plateau over a range of UTP substrate concentrations.

The Hanes-Woolf plots for CTPS 42, 44, and 46 (Figs. 2B, 4B, and 5B) are concave; a minimum ordinate value is reached for a particular substrate concentration and the ordinate values then increase with even lower substrate concentrations. These plots can also be used to determine the $K_{m \text { app }}$ values for each of the variants (Table I). There may be a correlation for several of the variants between the UTP substrate concentration corresponding to the minimum ordinate value on the Hanes-Woolf plot and the $K_{m \text { app }}$ value obtained from the Lineweaver-Burk plot. For example, for CTPS 44 the $K_{m}$ app 
A

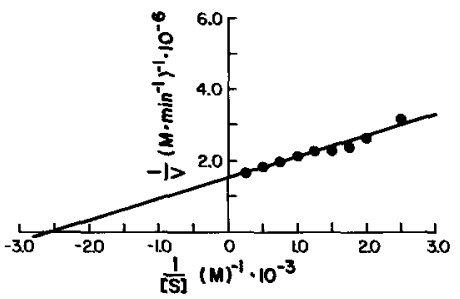

B
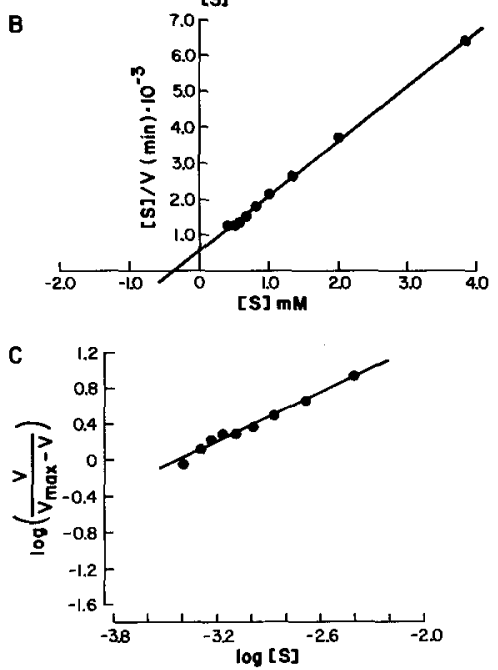

Fig. 1. Kinetic studies of CTP synthetase in Chinese hamster cell extracts. The cell extracts were V79 wild type. The graphical plots were (A) Lineweaver-Burk, $1 / v$ vs $1 / s$; (B) Hanes-Woolf, $s / v$ vs $s$; and (C) Hill, $\log \left[v /\left(v_{\max }-v\right)\right]$ vs $\log s$. Experimental conditions as described in the Materials and Methods of this paper and McLaren and Chu (1983).

A

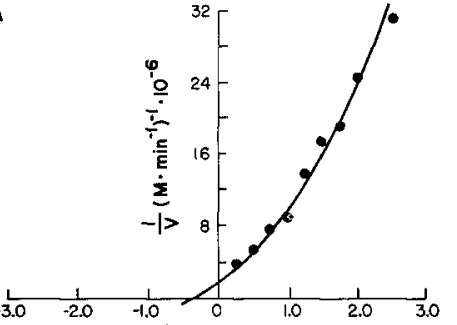

$\frac{1}{[\mathrm{~S}]}(\mathrm{M})^{-1} \cdot 10^{-3}$
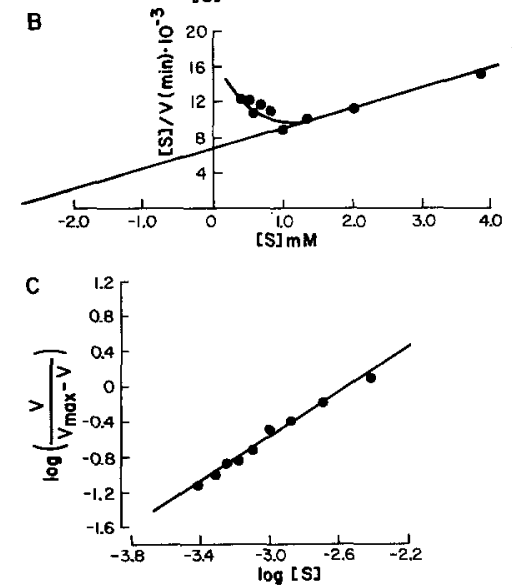

Fig. 2. CTPS 42. The graphical plots were (A) Lineweaver-Burk, $1 / v$ vs $1 / s$; (B) Hanes-Woolf, $s / v$ vs $s$; and (C) Hill, $\log \left[v /\left(v_{\max }-v\right]\right.$ vs $\log s$. Experimental conditions as described in the Materials and Methods of this paper and McLaren and Chu (1983). 
A

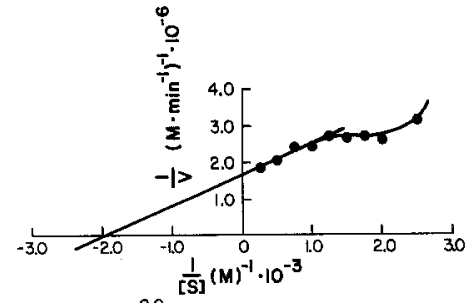

Fig. 3. CTPS 43. The graphical plots were (A) Lineweaver-Burk, $1 / v$ vs $1 / s$; (B) Hanes-Woolf, $s / v$ vs $s$; and (C) Hill, $\log \left[v /\left(v_{\max }-v\right]\right.$ vs $\log s$. Experimental conditions as described in the Materials and Methods of this paper and McLaren and Chu (1983).
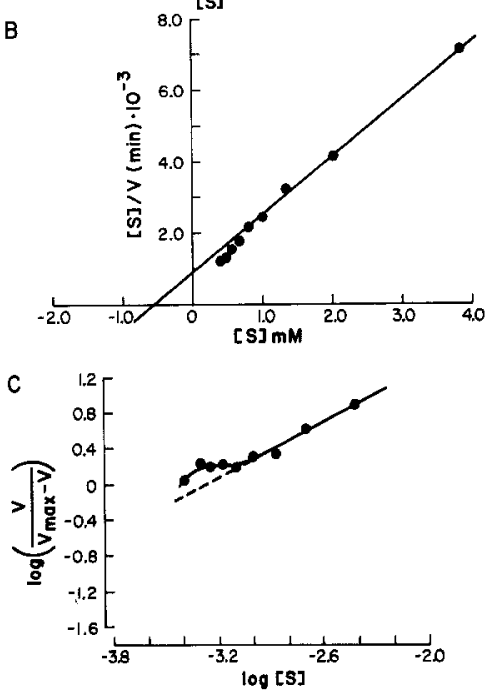

A

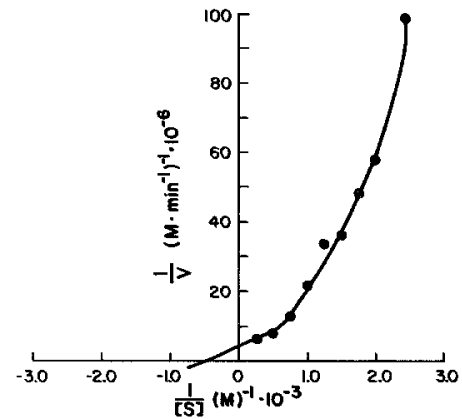

B
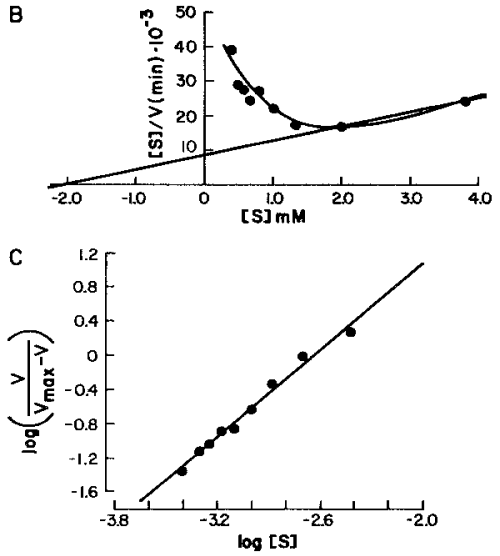

Fig. 4. CTPS 44. The graphical plots were (A) Lineweaver-Burk, $1 / v$ vs $1 / s$; (B) Hanes-Woolf, $s / v$ vs $s$; and (C) Hill, $\log \left[v /\left(v_{\max }-v\right]\right.$ vs $\log s$. Experimental conditions as described in the Materials and Methods of this paper and McLaren and Chu (1983). 
A

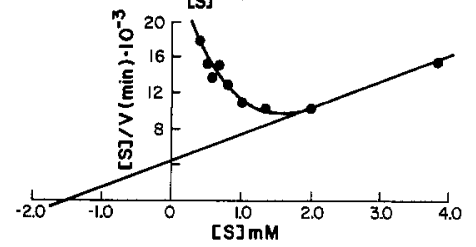

Fig. 5. CTPS 46. The graphical plots were (A) Lineweaver-Burk, $1 / v$ vs $1 / s$; (B) Hanes-Woolf, $s / v$ vs $s$; and (C) Hill, $\log \left[v /\left(v_{\max }-v\right]\right.$ vs $\log s$. Experimental conditions as described in the Materials and Methods of this paper and McLaren and Chu (1983).

from the Lineweaver-Burk plot is $2.1 \mathrm{~mm}$ and the minimum ordinate value from the Hanes-Woolf plot occurs at a UTP substrate concentration of about $2 \mathrm{mM}$ (Figs. 4A and B). The values for CTPS 46 are $K_{m \text { app }}=1.5 \mathrm{~mm}$ from the Lineweaver-Burk plot, and the minimum ordinate value from the HanesWoolf plot occurs at a UTP concentration of about $1.65 \mathrm{~mm}$ (Figs. 5A and B). There is less correlation for CTPS 42 , where the $K_{m \text { app }}$ is $3.0 \mathrm{mM}$ from the Lineweaver-Burk plot but the minimum ordinate value from the HanesWoolf plot occurs at a UTP concentration of about $1 \mathrm{mM}$ (Figs. 2A and B).

The reliability of the $K_{m}$ app values from the Hanes-Woolf plots depends on the number of experimental values that are used to construct a line of best fit. For example, the plot for the V79 cell extracts (Fig. 1B) indicates that a straight line can fit the data points and all points (nine) can be considered; the $K_{m \text { app }}$ from this plot would be reliable. On the other hand, the $K_{m \text { app }}$ values obtained for CTPS 42, 44, and 46 are all based on only two data points (Figs. $2 \mathrm{~B}, 4 \mathrm{~B}$, and $5 \mathrm{~B}$ ) and the reliability of these measurements is minimal.

The $K_{m \text { app }}$ and $V_{\max }$ values were determined from the Lineweaver-Burk plots by linear extrapolation using only the values corresponding to the higher substrate concentrations. However, since three variants exhibit curves that extrapolate close to the origin of the Lineweaver-Burk plots (Figs. 2A, 4A, and 5A), some latitude should be accorded these values. We have used the Lineweaver-Burk plots to determine $V_{\max }$ for all cell extracts, and the $V_{\max }$ 
Table I. Apparent Kinetic Constants of CTP Synthetase for UTP in V79 Cells and Mutant Derivatives

\begin{tabular}{|c|c|c|c|}
\hline Cell type & $\begin{array}{c}\text { Lineweaver-Burk plot, } \\
1 / v \text { vs } 1 / s\end{array}$ & $\begin{array}{c}\text { Hanes-Woolf plot, } \\
s / v \text { vs } s\end{array}$ & $\begin{array}{c}\text { Hill plot } \\
\log \left[v /\left(v_{\max }-v\right)\right] \text { vs } \log s\end{array}$ \\
\hline V79 & $K_{m \text { app }}=0.39 \mathrm{mM}^{a}$ & $K_{m \text { app }}=0.37 \mathrm{mM}$ & $\begin{aligned} S_{50} & =0.37 \mathrm{mM} \\
n_{\text {app }} & =0.92 \\
K^{\prime} & =0.70 \mathrm{mM}\end{aligned}$ \\
\hline CTPS 42 & $K_{m \text { app }}=3.0 \mathrm{mM}$ & $K_{m \text { app }}=3.0 \mathrm{mM}$ & $\begin{aligned} S_{50} & =2.8 \mathrm{mM} \\
n_{\mathrm{app}} & =1.3 \\
K^{\prime} & =0.56 \mathrm{mM}\end{aligned}$ \\
\hline CTPS 43 & $K_{m \mathrm{app}}=0.52 \mathrm{mM}$ & $K_{m \mathrm{app}}=0.55 \mathrm{mM}$ & $\begin{aligned} S_{50} & =0.54 \mathrm{mM} \\
n_{\mathrm{app}} & =1.1 \\
K^{\prime} & =0.35 \mathrm{mM}\end{aligned}$ \\
\hline CTPS 44 & $K_{m \text { app }}=2.1 \mathrm{mM}$ & $K_{m \text { app }}=2.1 \mathrm{mM}$ & $\begin{aligned} S_{50} & =2.3 \mathrm{mM} \\
n_{\mathrm{app}} & =1.7 \\
K^{\prime} & =3.4 \times 10^{-5} \mathrm{M}\end{aligned}$ \\
\hline CTPS 46 & $K_{m \text { app }}=1.5 \mathrm{mM}$ & $K_{m \text { app }}=1.5 \mathrm{mM}$ & $\begin{aligned} S_{50} & =1.9 \mathrm{mM} \\
n_{\mathrm{app}} & =1.7 \\
K^{\prime} & =2.9 \times 10^{-5} \mathrm{M}\end{aligned}$ \\
\hline
\end{tabular}

${ }^{a}$ The kinetic constants are pseudo $K_{m \text { app }}$. All values have been rounded off after calculation.

values were used to calculate the data points for the Hill plots. Each Hill plot allowed the determination of $S_{50}, n_{\mathrm{app}}$, and $K^{\prime}$. We find a good correlation between the $K_{m \text { app }}$ values determined from Lineweaver-Burk plots and the $S_{50}$ values determined from Hill plots (i.e., Table I and Figs. 1-5A and C). The $K_{m \text { app }}$ and $S_{50}$ values for CTPS 42,44 , and 46 are greater than the corresponding values for the V79 cells.

CTPS 43 has kinetic characteristics that appear to be different from those of either V79 cells or any of the other three variants. For example, the Lineweaver-Burk plot for CTPS 43 (Fig. 3A) appears to have a plateau region where the velocity of the reaction remains fairly constant over a range of UTP substrate concentrations. This pattern differs from V79, where the plot (Fig. 1A) is linear over the same substrate concentrations, and from the other three variants, where the plots (Figs. 2A, 4A, and 5A) are concave, indicating positive allosteric cooperativity. It is uncertain where a line should be drawn in Fig. 3A that would give the best values for $V_{\max }$ and $K_{m \text { app }}$. We have chosen the five data points representing the higher UTP substrate concentrations; the values obtained are $V_{\max }=0.60 \times 10^{-6} \mathrm{~mol} / \mathrm{liter} / \mathrm{min}$ and $K_{m \text { app }}=0.52 \mathrm{mM}$. The same problem also exists with the Hanes-Woolf plot (Fig. 3B). If the five data points were chosen representing the higher UTP substrate concentrations, the values obtained are $V_{\max }=0.61 \times 10^{-6}$ $\mathrm{mol} /$ liter $/ \mathrm{min}$ and $K_{m \text { app }}=0.55 \mathrm{mM}$. When the $V_{\max }$ obtained from the Lineweaver-Burk plot (Fig. 3A) is used to calculate the points for a Hill plot, the results obtained are shown in Fig. 3C. If the five data points representing 
the higher UTP substrate concentrations are used to draw the Hill plot, $S_{50}=$ $0.54 \mathrm{mM}, n_{\text {app }}=1.1$, and $K^{\prime}=0.35 \mathrm{mM}$ are obtained. The $K_{m \text { app }}, S_{50}$, and $n_{\text {app }}$ values for CTPS 43 are more like the values for V79 cells than the values of the other three variants. The only clear difference between V79 and CTPS 43 cells in these kinetic experiments is the velocity plateau for CTPS 43 . We do not believe that the small differences in $K_{m \text { app }}$ and $S_{50}$ between V79 and CTPS 43 can be considered significant in these experiments.

Figure 6A shows the results of experiments designed to measure the inhibition of CTP synthetase in vitro by adding CTP to assay tubes containing the extracts of the parental or variant cells. For each cell extract, CTP synthetase activity was measured at five different UTP concentrations, with or without added nonradioactive CTP at a fixed concentration $(0.37 \mathrm{mM})$. The results obtained from a V79 cell extract and graphed using the LineweaverBurk plot (Fig. 6A) show a marked sensitivity to added nonradioactive CTP. The inhibition of CTP synthetase increases with decreasing UTP concentra-

Fig. 6. Kinetic studies of CTP feedback inhibition of CTP synthetase in Chinese hamster cell extracts. The cell extracts were V79 (A) and CTPS 42 (B). Measurements were made at five different UTP concentrations, in the presence (filled symbols) or absence (open symbols) of 0.37 mM nonradioactive CTP. Experimental conditions as described in the Materials and Methods of this paper and McLaren and Chu (1983).

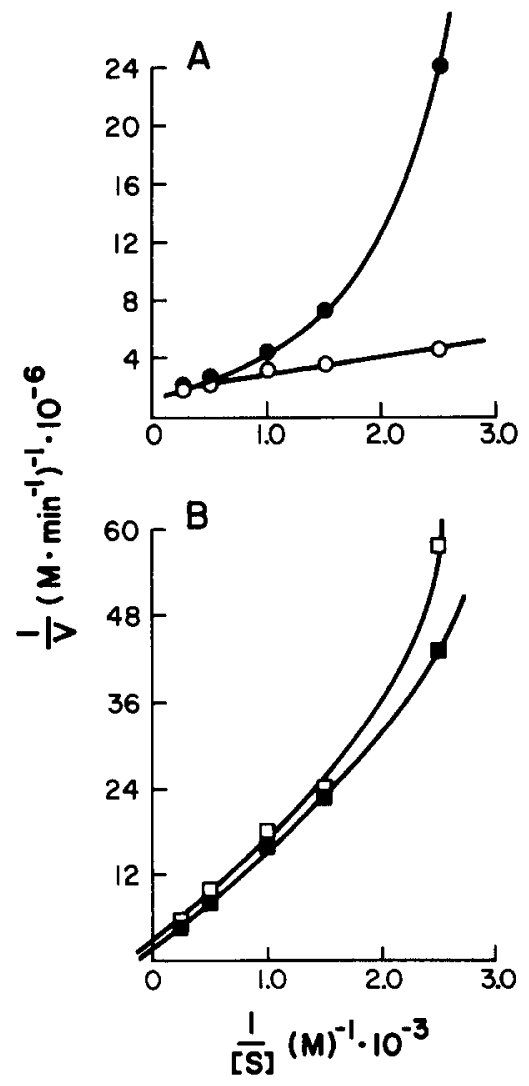


tions. At the lowest UTP concentration used $(0.40 \mathrm{mM})$, there is about an $80 \%$ reduction in CTP synthetase activity when approximately an equal amount of nonradioactive CTP $(0.37 \mathrm{mM})$ is added. In contrast, CTP synthetase from CTPS 42 (Fig. 6B) demonstrated virtually complete resistance to CTP inhibition. The plots for the other three variants are similar but are not presented here. Thus at each UTP concentration used, the conversion of UTP to CTP was not reduced by the addition of exogenous nonradioactive CTP. With all cell extracts examined (wild type and the four variants) we observed a concave Lineweaver-Burk plot when exogenous CTP was present, indicating that positive allosteric cooperativity is a component of the feedback inhibition.

\section{DISCUSSION}

The enzyme CTP synthetase catalyzes the following reaction:

$$
\begin{aligned}
\mathrm{UTP} & + \text { L-glutamine }\left(\text { or } \mathrm{NH}_{3}\right)+\mathrm{ATP} \underset{\mathrm{GTP}}{\stackrel{\mathrm{Mg}^{2+}}{\longrightarrow}} \mathrm{CTP} \\
& + \text { L-glutamic acid }+\mathrm{ADP}+\mathrm{Pi} .
\end{aligned}
$$

It is a complex enzyme with allosteric properties; details concerning the mechanism may be found in a review by Koshland and Levitzki (1974).

The limitations of the present study are the following: (1) some experiments were conducted only once; (2) where experiments were repeated the exact values were not always duplicated, but the results of repeat experiments are similar and appear to be satisfactory in demonstrating general trends; (3) the experiments focused on only one substrate, UTP; and (4) the experiments were conducted using crude cell extracts rather than purified enzyme preparations. This study specifically focuses on changes in $K_{m \text { app }}$ and $S_{50}$ of the substrate UTP assayed in various cell extracts. We have not examined the kinetics of other substrates, i.e., L-glutamine, and ATP. The apparent synergism between potential amino donors for V79 Chinese hamster cells, previously reported by this laboratory (McLaren and Chu, 1983), has been restricted to the potential amino donors $\mathrm{L}$-glutamine and $\mathrm{NH}_{3}\left(\mathrm{NH}_{4} \mathrm{HCO}_{3}\right)$ in this communication. Recent experiments, conducted after the kinetic studies presented here were completed, suggest that there is a carbonate and/or bicarbonate effect when $\mathrm{NH}_{4} \mathrm{HCO}_{3}$ is used. Thus, the apparent synergism observed between L-glutamine and $\mathrm{NH}_{4} \mathrm{HCO}_{3}$ may be due primarily to the $\mathrm{HCO}_{3}{ }^{-}$component of $\mathrm{NH}_{4} \mathrm{HCO}_{3}$. This carbonate/bicarbonate effect could account for the problems of exact reproducibility in the enzyme assays, since carbonate/bicarbonate may be present in varying amounts in the solutions 
used in the assay. Work is currently underway to elucidate further the details of the apparent synergism and the results will be presented elsewhere.

The use of cell extracts rather than purified enzyme preparations is based on two reasons. First, the variants had CTP synthetase activities that were generally lower than that of the V79 cell extracts. Since CTP synthetase activity can decrease with time after an extract is prepared, the enzyme activities in the variants were difficult to measure and some extracts had such low activity following their preparation that they were of little value. Second, enzyme purification is hindered by the difficulty of obtaining sufficient quantities of cultured fibroblasts.

Future work with the variants could include the following: (1) determination of $K_{m \text { app }}$ and $S_{50}$ for the other substrates, (2) determination of the ability of 3-deaza-UTP to compete with UTP for the UTP binding site, and (3) determination of the possible feedback inhibition by CTP analogues. However, more definitive biochemical characterization of the normal and altered CTP synthetase from these cells must depend on the availability of purified hamster enzymes, an attempt which is underway in this laboratory.

The key question to be answered at present is whether the observed differences in the enzyme kinetics and CTP feedback inhibition between cell extracts of the parent and those of the variant cells are consistent with the differences in their complex phenotypes. The four variant isolates were not auxotrophic for cytidine and would be expected to have sufficient CTP synthetase to allow growth in the absence of added cytidine; the variants did in fact have generally lower but measurable CTP synthetase activity. The variants selected were resistant to 3-deazauridine (DAU); DAU is metabolized by cells to 3-deaza-UTP, which has been demonstrated to inhibit mammalian CTP synthetase (McPartland et al., 1974; Brockman et al., 1975). Presumably 3-deaza-UTP occupies the UTP binding site on CTP synthetase and cannot be converted to 3-deaza-CTP; however, it prevents UTP from binding and thus prevents CTP formation. One explanation for DAU resistance would be that a mutation has occurred that alters the UTP binding site of CTP synthetase; the altered UTP binding site would have less affinity for 3-deaza-UTP and this would result in less inhibition. Such a change in the UTP binding site would most likely be reflected by a change in the $K_{m \text { app }}$ and $S_{50}$ for UTP in the variants. Our results indicate that the variants have higher $K_{m}$ app and $S_{50}$ values for UTP than the parental cells. The kinetic constants of the four variants also differ from each other (Table I). Additionally, the variants have elevated levels of cellular CTP and dCTP (Chu et al. 1984) and are more resistant to CTP feedback inhibition than the parental cells (this study). We interpret this to mean that an independent mutational event(s) in each of the four variant clones isolated has altered the binding of the substrate UTP. The mutational origin of the variants is 
consistent with, but not proven by, the fact that they arose in a cell population treated with a known chemical mutagen. The existence of mutations which alter the catalytic activity of CTP synthetase while allosterically modifying its regulation is in agreement with the finding that the control exerted by CTP on this enzyme activity takes place at a site distinct from the UTP binding site (McPartland and Weinfeld, 1979).

\section{ACKNOWLEDGMENTS}

The authors thank Barbara Lamb for technical assistance, Jules Shafer and Vincent Massey for suggestions, and Lisa Campeau for the preparation of the manuscript.

\section{REFERENCES}

Brockman, R. W., Shaddix, S. C., Williams, M., Nelson, J. A., Rose, L. M., and Schabel, F. M., Jr. (1975). The mechanism of action of 3-deazauridine in tumor cells sensitive and resistant to arabinosylcytosine. Ann. N.Y. Acad. Sci. 255:501.

Chu, E. H. Y., McLaren, J. D., Li, I. -C., and Lamb, B. (1984). Pleiotropic mutants of Chinese hamster cells with altered cytidine 5'-triphosphate synthetase. Biochem. Genet. 22:701.

Koshland, D. E., Jr., and Levitzki, A. (1974). CTP synthetase and related enzymes. In Boyer P. D. (ed.)., The Enzymes, Academic Press, New York, Vol. 10, pp. 539-559.

McLaren, J. D., and Chu, E. H. Y. (1983). Apparent synergism between amino donors for CTP synthesis in Chinese hamster fibroblasts. Mol. Cell. Biochem. 57:167.

McPartland, R. P., and Weinfeld, H. (1976). Cytidine 5'-triphosphate synthetase of calf liver-size, polymerization, and reaction stoichiometry. J. Biol. Chem. 251:4372.

McPartland, R. P., and Weinfeld, H. (1979). Cooperative effects of CTP on calf liver CTP synthetase. J. Biol. Chem. 254:11394.

McPartland, R. P., Wang, M. C., Bloch, A., and Weinfeld, H. (1974). Cytidine 5'-triphosphate synthetase as a target for inhibition by the antitumor agent 3-deazauridine. Cancer Res. 34:3107. 